This item was submitted to Loughborough's Research Repository by the author.

Items in Figshare are protected by copyright, with all rights reserved, unless otherwise indicated.

\title{
Electrospinning of natural polymers for advanced wound care: towards responsive and adaptive dressings
}

\section{PLEASE CITE THE PUBLISHED VERSION}

http://dx.doi.org/10.1039/c6tb00804f

\section{PUBLISHER}

(C) The Royal Society of Chemistry

\section{VERSION}

AM (Accepted Manuscript)

\section{PUBLISHER STATEMENT}

This work is made available according to the conditions of the Creative Commons Attribution-NonCommercialNoDerivatives 4.0 International (CC BY-NC-ND 4.0) licence. Full details of this licence are available at: https://creativecommons.org/licenses/by-nc-nd/4.0/

\section{LICENCE}

CC BY-NC-ND 4.0

\section{REPOSITORY RECORD}

Mele, Elisa. 2016. "Electrospinning of Natural Polymers for Advanced Wound Care: Towards Responsive and Adaptive Dressings". figshare. https://hdl.handle.net/2134/23186. 


\section{Journal of Materials Chemistry B}

\section{Electrospinning of natural polymers for advanced wound care: towards responsive and adaptive dressings}

Received 00th January 20xx Accepted 00th January 20xx

DOI: $10.1039 / \times 0 \times x 00000 x$

www.rsc.org/

\begin{abstract}
E. Mele ${ }^{\mathrm{a}}$
In the last years, the health-care services have registered worldwide an increased number of patients suffering from chronic wounds and ulcers that are mainly associated with diabetes, obesity and cancer. This has stimulated the research of advanced therapies for promoting the rapid and effective regeneration of the injured tissues. This review will discuss how biomimetic architectures produced by electrospinning natural biopolymers fulfil most of the requirements of ideal dressings. The recent progress in the area of portable electrospinning systems and of multiscale instructive materials that integrate stimuli responsive and sensing elements will be examined.
\end{abstract}

\section{Introduction}

Delayed or impaired wound healing is nowadays a global health issue that affects individuals suffering from diseases such as diabetes, obesity and cancer. ${ }^{1,2}$ Non-healing wounds have negative impact on patient quality of life, including pain, physical discomfort and psychological distress, as well as an worldwide economic burden of over 9.5 billion US dollars a year. ${ }^{3}$ Only in 2014, 6.0 and $9.3 \%$ of the UK and US population, respectively, were diagnosed with diabetes, ${ }^{4}$ and a global number of 439 million diabetic adults is estimated by $2030 .^{3}$ Nearly 600,000 hospitalisations every year are related to diabetic foot ulcers, ${ }^{1}$ whose common complications are infections, amputations (up to $15 \%$ of the patients) ${ }^{4}$ and mortality. Together with diabetes, the incidence of pressure and venous ulcerations is expected to rise in the next years as a consequence of both the aging population (up to $60 \%$ increase of Europe population aged above 65 by 2050) ${ }^{1}$ and the obesity ( $20 \%$ obese world's adults by 2030$).{ }^{5}$ Within this scenario, traditional therapies for wound management have been questioned and the need for advanced and more effective strategies has firmly emerged.

Relying on the better understanding of the cellular and molecular stages of the healing process and on the progress achieved in the area of biomaterials for tissue engineering, ${ }^{6-8}$ bioengineered wound dressings have been developed. ${ }^{9-11}$ Among them, fibrous nanostructures made of natural or

\footnotetext{
Department of Materials, Loughborough University, LE11 3TU, Loughborough (United Kingdom). E-mail: E.Mele2@lboro.ac.uk; Tel: +4401509 228595.

† Footnotes relating to the title and/or authors should appear here. Electronic Supplementary Information (ESI) available: [details of any supplementary information available should be included here]. See DOI: $10.1039 / x 0 x x 00000 x$
}

synthetic polymers and produced by electrostatic spinning have recently received great attention. ${ }^{12-14}$ These are in fact advanced and bioactive scaffolds that not only physically protect the wound from contaminants and infections like traditional dressings, but also are able to provide an ideal environment for skin regeneration, to release bioactive molecules, and to degrade at a controlled rate.

This review provides an overview of the recent developments in the area of nanofibrous wound dressings based on natural bio-macromolecules. First, the key aspects of the diverse phases of the healing process will be described and how their understanding at biochemical level has influenced the wound dressing market over the last years. Next, the electrospinning technique will be discussed in terms of methods suitable for producing fibres with controlled morphology and organisation, and miniaturised portable systems that find potential application in the wound care sector. Proteins (collagen, gelatine, fibrinogen and silk fibroin) and polysaccharides (hyaluronic acid, chitosan and alginate) derived from natural sources and used for fabricating biomimetic dressings will be examined, focusing on how their properties have been advantageously exploited for promoting skin regeneration. Finally, a perspective on the future trends will be given, considering strategies for the design and manufacture multiscale instructive, responsive and adaptable biomedical systems.

\section{Wound types, healing process and traditional dressings}

Adult skin is composed of two layers: the epidermis, which is the superficial stratified epithelium mainly consisting of keratinocytes, and the underlying dermis, which is a connective tissue rich in collagen. ${ }^{15,16}$ The role of the epidermis 
is to protect the human body from the external environment, whilst the dermis serves as mechanical support. A disruption of the normal anatomical structure and function of the skin is generally known as wound. ${ }^{10,17}$ It can be caused by mechanical, chemical or thermal traumas or be the result of specific medical problems.

According to the depth of the injured area, wounds are classified in: superficial wounds when only the epidermis is involved; partial-thickness wounds when the entire skin (epidermis, dermis, hair follicles, glands and hair follicles) is damaged; full-thickness wounds if subcutaneous tissues (muscles, tendons or bones) are also affected. ${ }^{10}$ Furthermore, depending on the time required for completing the healing process, wounds are divided in acute and chronic. Acute wounds are due to mechanical stresses (abrasions, lacerations, incisions and contusions), exposure to corrosive chemicals, heat, light and electrical shock. They usually heal completely within a period of 8-12 weeks. ${ }^{17}$ On the contrary, chronic wounds are consequence of diseases, such as vascular insufficiency (venous or arterial), pressure necrosis, prolonged inflammation, severe infections, cancer and diabetes. ${ }^{1}$ They require a healing time that exceeds the 12 weeks, and often fail to reach a normal healthy state, but instead persist in a pathologic condition of inflammation. The incomplete and delayed healing, together with the excessive production of exudates can even induce maceration of the not-injured skin nearby the wound. ${ }^{18}$

The development of appropriate dressings for the diverse types of wound requires knowledge on the intracellular and intercellular pathways that are activated during skin repair. ${ }^{6}$ As shown in Figure 1, fours sequential and overlapping phases characterise the classic wound healing model: homeostasis, inflammation, proliferation and remodelling. ${ }^{15,16,19}$ Homeostasis is the immediate response of the body to the injury (seconds-minutes) in order to stop blood loss at the wound site. A platelet plug is formed, followed by a fibrin clot that consists of a matrix of polymerised fibrinogen, fibronectin, vitronectin, and thrombospondin. ${ }^{18}$ The fibrin clot, whilst temporarily protects the wound from bacteria colonisation, acts as scaffold for the infiltration of inflammatory cells, such as neutrophils and monocytes. During the inflammation phase (hours-days), neutrophils are recruited in response to plateletproduced cytokines, and they remove foreign particles, dead cells and bacteria. Subsequently, monocytes, which differentiate in macrophages, are involved in the debris clearance, but also in the production of cytokines and growth factors that stimulate the migration of fibroblasts and epithelial cells to the wound. ${ }^{15,20}$ Those cells, together with keratinocytes, are essential in the proliferation phase (daysweeks) that is characterised by re-epithelialisation, angiogenesis and formation of proteins of the extracellular matrix (ECM), such as collagens, fibronectin, and vitronectin. Granulation tissue replaces the fibrin clot and fibroblasts tend to differentiate in myofibroblasts, which are contractile cells able to promote

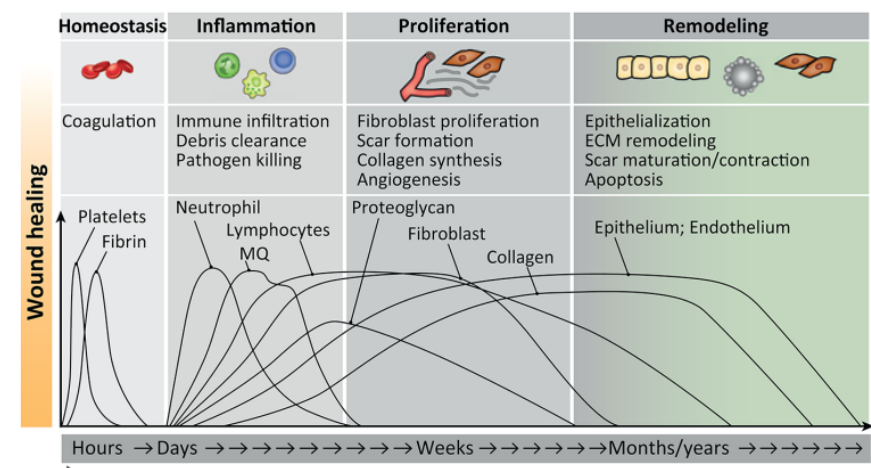

\& Injury

Figure 1. The time line of the four sequential and overlapping phases for the model of wound healing: homeostasis, inflammation, proliferation and remodelling. Adapted from ref. 19 with permission from Elsevier.

closure and remodelling of the wound. During the remodelling stage (from weeks to years), wound contraction and apoptosis of most of the endothelial cells, macrophages and myofibroblasts take place, resulting in the formation of acellular scar tissue, maturation of ECM proteins and increased organisation of collagen fibrils. ${ }^{6,13}$ After this phase, the skin is completely regenerated and its functionalities are restored. On the contrary, in the case of chronic wounds the healing cascade is impaired, for instance as consequence of dysfunctional behaviour of neutrophils and macrophages, or imbalance of ECM deposition. ${ }^{18}$

\subsection{Selection of commercially available dressings}

Despite the intense research efforts in the field of wound management, an ideal therapy suitable for promoting rapid and efficient healing has not been yet developed. Currently, most of the commercially available dressings are natural or synthetic gauzes and bandages that play only a passive role in the healing process. ${ }^{10}$ When used as primary dressings (in direct contact with the wound), they simply protect the injured site from contaminations and absorb exudates in a noncontrolled way. This determines dehydration of the wound site and increased adhesion of the bandage to it, with consequent healing delay and patient discomfort and pain. ${ }_{17}$ On the other hand, traditional bandages can be used as secondary dressings (as cover of primary ones) in combination with moistureretentive and antibacterial-impregnated systems. ${ }^{21}$

Moisture-retentive dressings include films, hydrogels, hydrocolloids and foams. Semi-permeable and transparent films are typically indicated for superficial and lightly exudative wounds, and first-degree burns. They are permeable to both water vapour and oxygen, provide a barrier against microorganisms, and allow visual checks of the wound status. Commercial examples are flexible polyurethane membranes, such as Bioclusive (Systagenix), Tegaderm (3M) and Opsite (Smith \& Nephew). ${ }^{22,23}$ Instead, dry and necrotic ulcers can be treated with hydrogel-based dressings (water content higher than $90 \%$ ) that supply moisture, rehydrating the tissue and reducing the local temperature. However, limitations of these dressings are poor mechanical properties and risk of skin maceration. Natural materials, such as carboxymethylcellulose 
(CMC, IntraSite by Smith \& Nephew), hydroxyethylcellulose (HEC, Nu-Gel by Johnson \& Johnson), starch (Aquaform, Unomedical), pectin (Granu-Gel, ConvaTec) and alginate (Tegagen, $3 \mathrm{M}$ ), are mainly used in the industrial production of hydrogels. ${ }^{24}$ Among them, alginate, a polysaccharides derived from seaweeds (as will be discussed in further detail in Section 4.6) exhibits attractive properties also as hydrocolloid dressing.

Hydrocolloids are indicated for deep and exudative ulcers, and partial-thickness wounds. They are able to absorb physiological fluids and form gels that provide thermal insulation and protection against bacterial colonisation. ${ }^{25-27}$ Well-known brands include Comfeel by Coloplast (CMC and calcium alginate), Cutinova Hydro by Smith \& Nephew (hydrophilic polyurethane matrix containing particles of superabsorbent polyacrylate), DuoDerm by CovaTec (mix of CMC, gelatin and pectin), Seasorb by Coloplast (freeze dried calcium/sodium alginate). Materials for hydrocolloid dressings can be processed in different forms: sheets, fibres, beads and foams. In particular, foams, such as PolyMem (Aspen medical Europe) and Cavi-Care (Smith \& Nephew), are made of polyurethane and silicone. ${ }^{26}$ They are characterised by surfaces with distinctive wetting behaviour: an hydrophilic surface in contact with the wound to promote the absorption of exudates, and an hydrophobic opposite layer to avoid loss of fluids. The porosity of the foams facilitates the removal of liquids from the wound site, whilst maintaining a moist environment and allowing breathability. Furthermore, thermal insulation and comfort are ensured. However, the chemical composition of the foams can induce allergic reactions in sensitive individuals with a consequent inflammation response.

Infections associated with bacteria and fungi have a detrimental effect on the healing process, particularly in the case of burns and chronic wounds. ${ }^{21}$ Therefore, antimicrobialimpregnated dressings have been proposed. Silver ions and nanoparticles are widely accepted for their topical antiseptic activity, and they can be incorporated in foams (PolyMem Silver by Aspen Medical Europe, GranuFoam Silver by Kinetic Concepts) or used to functionalise meshes (Acticoat by Smith \& Nephew, Sorbsan Silver by Aspen Medical Europe). ${ }^{28,29}$ For superficially infected wounds, iodine (Inadine by Systagenix) or natural ingredients, such as medical grade honey (Activon Tulle by Advancis Medical), polysaccharides (chitosan), plant extracts and essential oils, are valid alternative as well. ${ }^{30}$ Evidences show that solid dressings are to be preferred to liquid or gel-like formulations (ointments, creams, emulsions), because the latter are prone to modify their rheological properties due to the presence of exudates, and therefore have a limited activity. ${ }^{10}$

Although a wide and diversified range of wound dressings is currently available, the functions that even the most advanced system can accomplish are still limited and only slightly improved with respect to the traditional counterparts: physical protection, oxygen permeation, moist environment and prevention of microorganism colonisation. Because the main aim of wound care is to heal the injured tissue in the most effective and rapid way, an ideal dressing should have an influence on the cellular pathways of tissue regeneration. Therefore, key desiderata are: a bio-inspired architecture that resembles the natural ECM organisation in order to support cell adhesion, proliferation and differentiation; the delivery of drugs and growth factors to accelerate tissue growth; the controlled biodegradation of the scaffold to avoid further traumas during dressing detachment and peeling.

The current research in the sector has pointed out that nanostructured meshes produced by electrostatic spinning (ES) are suitable for the development of the new class of multifunctional and bioactive dressings. ${ }^{13,14,31-33}$ Particular attention has been devoted to the electrospinning of materials derived from natural resources, thanks to their similarity to bio-macromolecules, ${ }^{12,34}$ as it will be discussed in the following sections.

\section{Biomimetic architectures by electrospinning}

Electrospinning is an electro-hydrodynamic process that allows the production of polymer fibres with a diameter ranging from few nanometres to few microns. ${ }^{35-37}$ As shown in Figure $2 a$, a classical electrospinning apparatus is composed of a spinneret (metallic needle connected to a syringe), a grounded metallic planar collector and a high voltage power supply. ${ }^{38}$ Solutions of polymers in a proper solvent or blend of solvents are injected through the needle, typically using a syringe pump in order to control the solution flow rate. When high voltage (of the order of 5 to $30 \mathrm{kV}$ ) is applied to the needle through an electrode, the surface charge density of the liquid droplet at the tip of the needle increases, with the consequent formation of a cone-like shape (Taylor cone). ${ }^{39}$ If the electric field applied overcomes the liquid surface tension, a filament of solution is ejected from the tip of the Taylor cone and it moves towards the collector. ${ }^{35}$ This stage of the electrospinning process is known as jet initiation. The electrically charged filament travels along a straight line for few centimetres and then experiences bending instability with the formation of a series of spiralling loops. ${ }^{40}$ During this phase, the filament elongates, reducing its diameter, and the solvent evaporates. Thus, the solidification of the filament takes place and a network of randomly distributed non-woven fibres is collected on the grounded target (Figure $2 \mathrm{~b}$ and $2 \mathrm{c}$ ). ${ }^{41,42}$

Thanks to its high flexibility in terms of materials that can be processed and types of fibrous scaffolds that can be produced, the electrospinning technology has attracted enormous research and industrial interests in the last years. Natural and synthetic polymers, their blends and nano-composites can be easily structured by ES, resulting in fibres with engineered physicochemical properties. For instance, the diameter of electrospun fibres and consequently the porosity of the resulting mat can be controlled by adjusting the rheological properties of the solution (concentration, viscosity, electrical conductivity) and the operational parameters (voltage, 
(b)

(a)

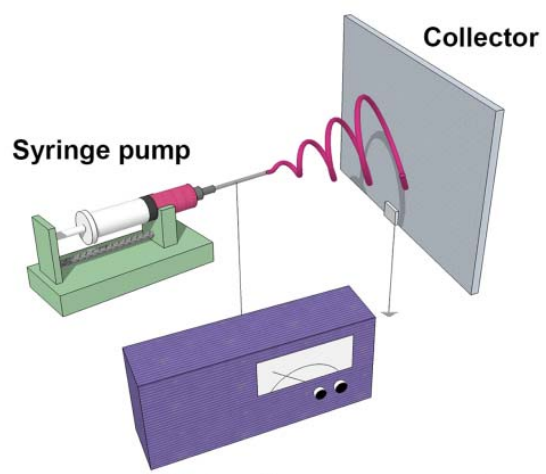

High voltage power supply

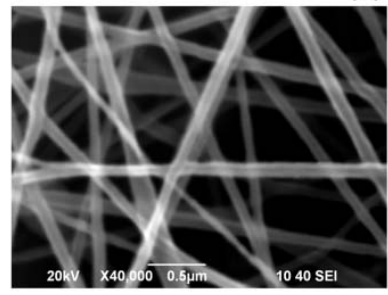

Diameter $\approx 80 \mathrm{~nm}$

(d)

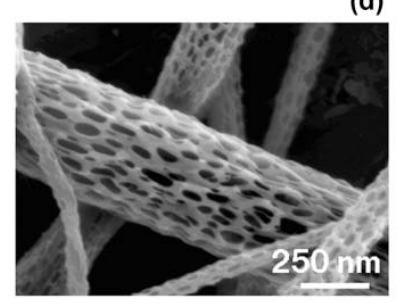

Porous fibres (c)

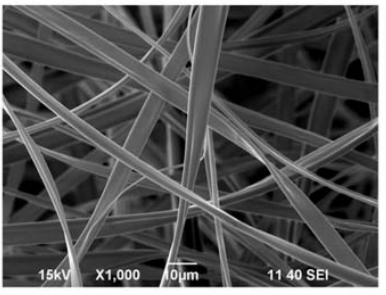

Diameter $\approx 4 \mu \mathrm{m}$

(e)

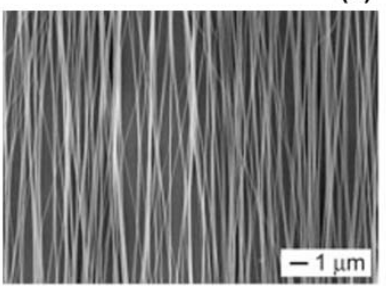

Aligned fibres

Figure 2: (a) Schematic representation of a conventional electrospinning apparatus consisting of a syringe pump, a high voltage power supply and a metallic planar collector. Fibres electrospun using this configuration are randomly distributed. Scanning electron microscope (SEM) images of (b) alginate and (c) cellulose acetate electrospun fibres having a diameter of approximately $80 \mathrm{~nm}$ and $4 \mu \mathrm{m}$, respectively. (b) is reproduced with permission from ref. 41. (c) is reproduced with permission from ref. 42. (d) SEM image of porous PLGA nanofibers. Reproduced with permission from ref. 44 . (e) SEM picture of uniaxially aligned fibres based on polyvinylpyrrolidone. Reproduced with permission from ref.46

injection rate, needle-collector distance, environmental temperature and humidity). ${ }^{32}$ Furthermore, multicomponent fibres and hierarchical architectures (fibres decorated with pores, papillae or knots) can be generated by properly designing ES apparatuses (Figure 2d). ${ }^{43,44}$ Modifications have been made at level of the spinneret and/or the collecting system. In the first case, an example of modified ES process is coaxial electrospinning, where multiple spinnerets are used to produce core-shell structures or multichannel nanotubes. ${ }^{45}$ In the second case, collectors (disks, cylinders, patterned elements) that can rotate at specific speed offer the possibility to achieve a good degree of alignment of the electrospun fibres (Figure 2e). ${ }^{32,46}$

High surface-to-volume ratio, porosity and threedimensional (3D) organisation make electrospun mats relevant for diverse applications, such as filtration, sensors, energy and catalysis; ${ }^{43,47,48}$ but it is for tissue engineering that those characteristics become crucial. Scaffolds produced by ES are capable to mimic the structure and physiology of the native ECM, which is in fact formed of a fibrillary network of proteins and polysaccharides, such collagen, hyaluronic acid and elastin. This biomimetic architecture provides topographical and biochemical cues to regulate cell behaviour and hence stimulate tissue regeneration ${ }^{49,50}$ In the specific instance of wound care applications, besides as scaffolding and instructive materials, electrospun nanofibers work also as exudate absorbers, membranes for oxygen and vapour permeation, barriers against microbial colonisation, systems for diffusion of nutrients and growth factors, and for delivery of drugs. ${ }^{12}$

\subsection{Portable electrospinning systems}

As discussed above, the properties of electrospun dressings are promising for the development of advanced therapeutic strategies for the treatment of acute and chronic wounds. For this reason, in order to make electrospinning even more accessible, ready to use in hospitals and clinical centres, and to target personalised healthcare, portable ES platforms have been proposed. ${ }^{51-55}$ Recently, Y.-Z. Long and collaborators have shown the in situ deposition of electrospun fibres using a battery-operated handheld electrospinning device that provides working voltages up to $10 \mathrm{kV} .^{52}$ The entire apparatus has a volume of $10.5 \times 5.0 \times 3.0 \mathrm{~cm}^{3}$ and a weight of $120 \mathrm{~g}$ (Figure 3a). They demonstrated that diverse biopolymers can be electrospun, including poly-caprolactone $(\mathrm{PCL})$ and poly(lactic acid) (PLA), even directly on human hands (Figure $3 b$ and $3 c)$. The fibres produced had a well-defined morphology, without defects, and a diameter ranging from 0.2 to $2.0 \mu \mathrm{m}$. This device has been used also for the deposition of antibacterial dressings ( $\mathrm{PCL}$ and $\mathrm{Ag}$ nanoparticles) on skin wounds in animal tests, showing the healing efficacy of the fibres. ${ }^{53}$ Other portable ES systems have been designed and manufactured by the same group, replacing the classical voltage power supply with hand-operated generators that can be self-powered or connected to solar cells. ${ }^{54,55} \mathrm{~A}$ further advance in the area of portable ES systems has been anticipated by P.-A. Mounthuy et al. with a pen extension device for controlling spatial deposition of the fibres during electrospinning. ${ }^{56}$

The main application envisaged for miniaturised and portable ES apparatuses is in wound care. Differently from the traditional benchtop ES, they can be operated by non- 

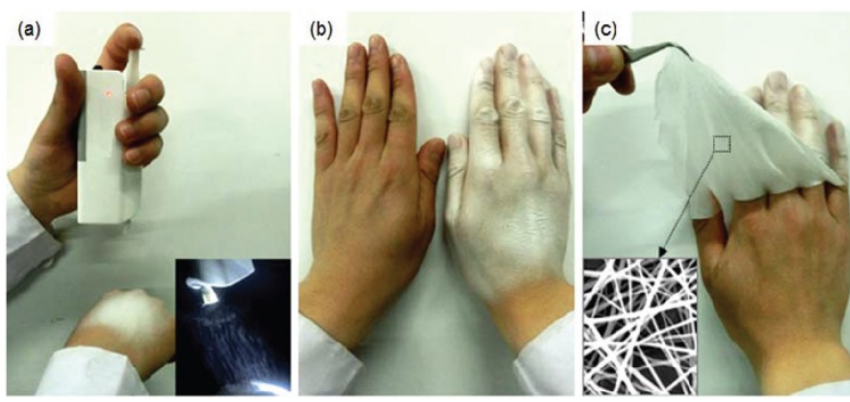

Figure 3. Photographs of the electrospinning process conducted with portable apparatus. (a) The device is used to deposit PLA fibres on the hand of the operator; (b) a uniform fibrous mat is obtained over the entire surface of the hand; (c) the electrospun mat can be easily removed using tweezers. Reproduced with permission from ref. 52 .

specialised users and in emergency situations. Because the dressing can be produced in real time, its chemical composition and bioactivity can be chosen according to the status of the wound and the patient needs. Moreover, the in situ deposition ensures good adhesion of the electrospun mat to the injured area, even on curved surfaces.

\section{Fibrous dressings based on natural polymers}

Naturally occurring proteins and polysaccharides have been extensively used in the production of electrospun nanofibrous scaffolds for wound management. ${ }^{12,34}$ The major motivation is to achieve the highest possible level of biomimicry, recreating the native ECM. Moreover, these natural macromolecules are biocompatible and biodegradable, and some of them possess also intrinsic antibacterial and anti-inflammatory properties. As expected, proteins that form fibres in nature, such as collagen, fibrinogen and silk fibroin, are extremely indicated for ES, and during the process they easily self-assemble in fibrous structures. ${ }^{57}$ On the other hand, the ES of polysaccharides, such as chitosan, cellulose and alginate, can present some challenges and in most of the cases additives are required to promote it. ${ }^{58}$

In this section, the natural polymers most utilized in wound healing will be discussed. They are classified in Scheme 1 according to their origin, and the principal sources of extraction.

\subsection{Collagen and gelatine}

Collagen is the principal structural component of the ECM and the most abundant protein in humans and animals. ${ }^{59}$ Its role is to provide mechanical strength to tissues and to stimulate cell adhesion and proliferation. ${ }^{60}$ Among the different types of collagen, type I constitutes $70-80 \%$ of the dermis, where it is organised in form of interwoven, randomly oriented bundles of fibrils. ${ }^{59}$ Type II and type III are present in minor amount in the skin, but are instead found in cartilage and blood vessels. ${ }^{12}$ During the wound healing process, fibroblasts produce collagen molecules that then aggregate to form fibrils having a diameter in the range of $10-500 \mathrm{~nm} .{ }^{61}$ This fibrous network facilitates cell migration to the wound site (endothelial cells, keratinocytes) and therefore has a crucial role in tissue repair. $^{62}$

Collagen can be electrospun from solutions based on fluoroalcohols, such as 1,1,1,3,3,3-hexafluoro-2-propanol (HFIP) and 2,2,2-trifluoroethanol (TFE), ${ }^{63}$ and water-ethanol mixtures. ${ }^{64}$ Although concerns have been arisen on the safety and possible effects of HFIP on collagen denaturation, it remains the solvent of choice. ${ }^{65}$ Collagen nanofibers have poor mechanical resistance and high degradation rate, therefore cross-linking procedures are required. Different methods have been indicated, after or during ES, such as stabilisation with glutaraldehyde, epoxy compounds, 1-ethyl-3-(3dimethylaminopropyl) carbodiimide, and exposure to ultraviolet light. ${ }^{65-67}$ The effect of cross-linked collagen nanofibrous membranes on the treatment of open wounds has been investigated, showing an improvement of skin healing due to high attachment and spreading of keratinocytes, and reduction of wound contraction. ${ }^{68,69}$

The demand of collagen dressings with better mechanical properties and improved functionalities has driven the research of composite alternatives, where collagen has been co-electrospun with synthetic or natural biopolymers. It has been demonstrated that collagen-PCL membranes possess enhanced physicochemical properties and they induce a better proliferation of fibroblasts and keratinocytes. ${ }^{70-72}$ Collagen and poly-D-L-lactide-glycolide (PLGA) scaffolds loaded with Glucophage, an anti-diabetic drug, were effective in the treatment of diabetic wounds in animal model, resulting in a rapid re-epithelialization of the skin and increased production of collagen. ${ }^{73}$ Combinations of collagen with other natural materials will be discussed in following sections.

A derivative of collagen that is highly used for biomedical applications is gelatine. It can be electrospun from solutions of HFIP, TFE, acetic and formic acid, ${ }^{74,75}$ and subsequently crosslinked, like collagen. Gelatin fibres that contain silver nanoparticles have been proposed for infected wounds. ${ }^{76,77}$ The release of silver ions from the mats is indeed functional in inhibiting the growth of bacteria, such as Pseudomonas aeroginosa, Staphylococcus aureus, Escherichia coli, and methicillin-resistant $S$. aureus. It has also been shown that the incorporation of Centella asiatica, a traditional herbal medicine, into gelatine and poly-vinyl alcohol (PVA) fibres is useful for promoting fibroblast proliferation and collagen synthesis in skin wound, whilst expressing antibacterial activity. ${ }^{78}$ Animals treated with the composite membrane presented a high recovery rate. PCL, polyurethane, and PLA have been also blended with gelatine, and composite dressings have been electrospun. ${ }^{79-81}$

\subsection{Hyaluronic acid}

Hyaluronic acid (HA) is a linear polysaccharide composed of alternating (1-4)- $\beta$ linked D-glucuronic and (1-3)- $\beta$ linked $N$ acetyl-D-glucosamine residues. ${ }^{82}$ This glycosaminoglycan (GAG) is a component of the ECM of connective tissues. HA is involved in the different phases of wound healing, activating and moderating the inflammatory response, facilitating 
Bio-macromolecules

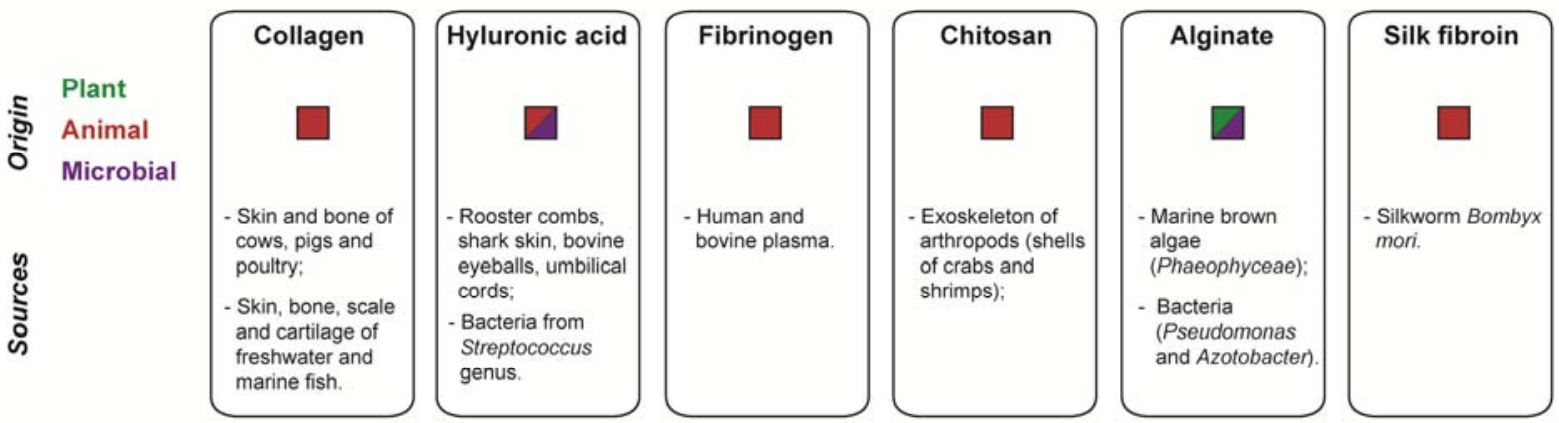

Scheme 1: Classification of natural proteins and polysaccharides used for electrospun wound dressings: origin and main sources of extraction.

keratinocytes migration and proliferation, and reducing scar formation. ${ }^{83}$ The high molecular weight (from 100 to 8000 $\mathrm{kDa})^{84}$ and hygroscopic nature of $\mathrm{HA}$ make it an ideal candidate for the production of hydrogels; but, on the other hand, the resulting high viscosity poses some limitations to the electrospinning process. ${ }^{85}$ Consequently, HA is often blended with other polymers or dissolved in appropriate solvent mixtures in order to modify its viscosity and promote the formation of fibres. ${ }^{86,87}$

Y. Qian at al. produced PCL/HA nanofibrous scaffolds, where $\mathrm{PCL}$ and HA offered, respectively, mechanical stability and biochemical cues. ${ }^{88}$ Enhanced fibroblasts infiltration and motility were observed both in vitro and in vivo for PCL/HA membranes if compared with PCL ones. This was possibly due to the interaction between $\mathrm{HA}$ and cell surface receptors. After 4 weeks post-implantation, histological analysis revealed that the penetration of cells was higher in scaffolds containing HA. HA has been proposed also in combination with collagen, ${ }^{89}$ resulting in electrospun dressings that exhibited an active role in cell secretion of proteinases and tissue inhibitors of metalloproteinases, and in reducing scar formation.

\subsection{Fibrinogen}

Fibrinogen is a protein present in the blood and it is involved in the haemostasis phase of wound healing, when it is converted in insoluble fibrin fibres. ${ }^{90}$ The electrospinning of fibrinogen has been first reported by G. E. Wnek and co-workers using a mixture of HFIP and minimal essential medium, as solvent. ${ }^{91}$ The resulting nanofibers had a diameter in the range of 80-700 $\mathrm{nm}$. Another study showed that single fibres electrospun following this protocol were characterised by elasticity and extensibility higher than collagen fibres. ${ }^{92}$ For biomedical applications, the degradation rate and mechanical resistance of fibrinogen scaffolds have been controlled either by crosslinking them with chemical compounds, such as glutaraldehyde vapour, or supplementing the cell culture medium with aprotin. ${ }^{93}$ Electrospun bandages of thrombin and fibrinogen have been proposed for full-thickness skin lesions in swine model, thanks to their haemostatic and antiinflammatory activity. ${ }^{94}$

\subsection{Chitosan}

Chitosan is a polysaccharides composed of glucosamine and $\mathrm{N}$ acetyl glucosamine units linked by (1-4) glycosidic bonds. ${ }^{95}$ It is obtained by the deacetylation of chitin, which is the second most abundant polysaccharide in nature (after cellulose) and the major structural component of the exoskeleton of shrimps and crabs, and of the cell walls of fungi. ${ }^{96}$ The ES of chitosan requires the use of acidic solutions, such as diluted hydrochloric acid, acetic acid, formic acid and trifluoroacetic acid (TFA), and of polymeric additives. ${ }^{96}$ The resulting scaffolds are characterised by haemostatic and antibacterial properties, low immunogenicity and biocompatibility, justifying the great interest in applying them for the treatment of wounds. Because of the large number of scientific papers published on electrospun chitosan dressings, this section will focus on the latest developments in the field.

Recently, composite chitosan fibres with enhanced antibacterial properties have been prepared using natural bioactive compounds, such as essential oils and honey. ${ }^{97-100}$ Cinnamon oil has been incorporated into chitosan/poly(ethylene oxide) (PEO) fibres having a diameter of around $50 \mathrm{~nm} .{ }^{97}$ The electrospun mats were able to release the essential oil in vitro, and exhibited antibacterial activity against $E$. coli and $P$. aeruginosa. In another work, extracts of Garcinia mangostana (GM), a tropical fruit, were added to chitosan-ethylenediaminetetraacetic acid/polyvinyl alcohol (CS-EDTA/PVA) solutions and electrospun. ${ }^{98}$ The scaffolds possessed antioxidant and antibacterial activity (E. coli and $S$. aureus), and were effective in accelerating wound healing in rats, with a wound closure rate higher than commercial antibacterial dressings. Sarhan et al. have produced honey/chitosan/PVA fibres loaded with Cleome droserifolia (CE) and Allium sativum extracts. ${ }^{99}$ Together with the ability to stop the formation of $S$. aureus biofilms, the composite dressings demonstrated high levels of fibroblasts 

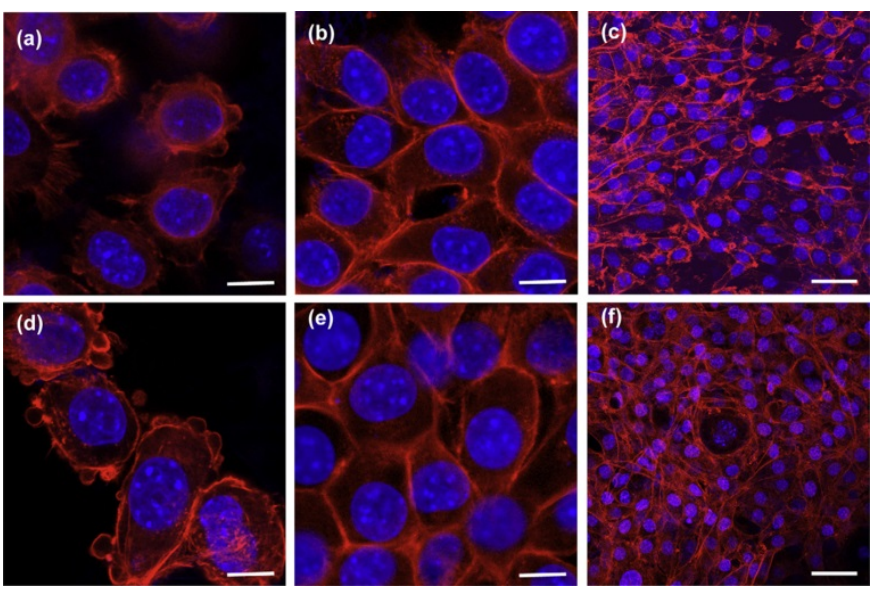

Figure 4: Images by confocal laser scanning microscopy of fibroblasts cultured on (a-c) PHBV/Chitosan (2:3 ratio) and (d-f) PHBV/Chitosan (4:1 ratio), for (a, d) 12 hours, (b, e) 2 days and (c, f) 7 days of seeding. F-actin and nucleus are stained in red and blue, respectively. Scale bar $=10 \mu \mathrm{m}$ (a, b, d, and e) and $50 \mu \mathrm{m}$ (c, f). After 12 hours, fibroblasts adhere and spread on the scaffolds, reaching the confluent state after 7 days. Reproduced with permission from ref. 101.

viability and enhanced wound repair in vivo, when compared with the commercial product Aquacel.

Hybrid and multilayers systems have been also developed for promoting cells proliferation and healing. ${ }^{101,102}$ For instance, poly(3-hydroxybutyrate-co-3-hydroxyvalerate) (PHBV) and chitosan mats have been tested as biocompatible scaffolds for skin regeneration (Figure 4). ${ }^{101}$ In vivo studies on full-thickness wound model demonstrated that the scaffolds absorbed the biological liquids, while decreasing inflammation and accelerating the proliferative phase. The wounds treated with the chitosan-PHBV scaffolds completely re-epithelized after 21 days, showing a high level of tissue organisation, angiogenesis and collagen fibres arrangement. Hierarchical nano/microfibrous chitosan/collagen systems have been produced by electrospinning chitosan followed by impregnation of the fibres with collagen solution and freezedrying. ${ }^{102}$ Fibroblasts and keratinocytes were cultured on the scaffolds, and ex vivo human skin equivalent model showed cells migration and wound re-epithelisation.

\subsection{Alginate}

Alginate is a polysaccharide abundant in brown algae and produced by some bacteria. ${ }^{103}$ It is constituted of $\alpha$-L-guluronic (G) and 1,4 linked- $\beta$-D-mannuronic (M) acid residues, arranged in a blockwise pattern with homopolymeric regions of $M$ and $G$ interspersed with regions of alternating MG units. The sodium salt of alginate is water soluble and it forms high viscous solutions even at very low polymer concentrations (2-3 wt\%). ${ }^{104}$ The electrospinning of those solutions can be carried out only in presence of synthetic polymers, such as PEO and PVA, which are able to reduce the repulsive force among the polyanionic alginate chains. ${ }^{12}$ However, the high solubility of the resulting mats limit their stability in aqueous environments, and consequently, for specific biomedical applications, cross-linking approaches are necessary (glutaraldehyde, divalent ions and TFA). ${ }^{105-107}$

Sodium alginate/PVA electrospun mats with antibacterial properties have been prepared using $\mathrm{ZnO}$ nanoparticles. ${ }^{108}$ The study showed that a right concentration of $\mathrm{ZnO}$ nanoparticles (from 0.5 to $5 \%$ ) should be selected in order to have nanocomposite fibres with antibacterial activity (against $S$. aureus and E. coli) and minimum cytotoxicity. In fact, if on one hand high $\mathrm{ZnO}$ concentrations are more effective against bacterial growth; on the other one they decrease fibroblasts viability. Therapeutic cargo (Lidocaine, Neomycin and Papain) have been also combined with alginate-PVA, in order to produce dressings that can prevent wound scarring. ${ }^{109}$ Moreover, alginate-PVA nanofibers have been proposed for the transdermal delivery of ciprofloxacin, an antibiotic drug. ${ }^{110}$ In vivo studies on rabbit wound model demonstrated that a sustained and controlled release of the drug can be obtained from the electrospun patches, positively affecting the wound healing rate. For the cure of UV-induced skin burns, instead, nanofibers of alginate-PEO and lavender essential oil have been used. $^{41}$ The composite mats exhibited antibacterial activity against $S$. aureus and anti-inflammatory properties, being able to reduce the production of pro-inflammatory cytokines both in vitro and in vivo. The skin of the treated animals recovered faster than in the case of untreated ones, and no appearance of erythema was detected.

\subsection{Silk fibroin}

Silk fibroin is a fibrous protein produced by some insects, such as the silkworm Bombyx mori. ${ }^{111}$ Because in nature it is protected by a coat of sericin (a glue-like protein), degumming procedures of raw silk fibres are required to make this protein available. It is well known that fibroin possesses unique properties for skin regeneration, including excellent biocompatibility, enhanced biosynthesis of collagen, reepithelialization, elimination of scarring, minimal immunogecity, hemostatic and anti-inflammatory activity. ${ }^{112}$ Therefore, attention has been directed in processing fibroin with electrospinning, with the aim to create bioactive dressings. ${ }^{113}$ 
Recently, elecrospun silk fibroin has been applied in burn rat model. ${ }^{114}$ Histological results showed that the scaffolds exhibited healing effect, increasing the deposition of collagen in the injured area, and significantly reducing the expression level of pro-inflammatory cytokines. Y.-H. Shan et al. have investigated the effect of fibroin-gelatine nanofibers loaded with astragaloside IV (a natural anti-inflammatory and antioxidant compound) on partial-thickness burn wounds. ${ }^{115}$ They observed that the composite scaffolds promoted fibroblasts adhesion and proliferation in vitro, and accelerated wound healing in vivo by reducing scar formation and increasing collagen organisation and angiogenesis. Other natural ingredients that have been used together with silk fibroin are Vitamin C and grape seed extract. ${ }^{112,116}$ The resulting fibres significantly enhanced the proliferation of fibroblasts, and protected them against oxidative stress. Hybrid scaffolds made of fibroin and synthetic or natural biopolymers, such as PLGA, chitosan and cellulose, have been also developed. ${ }^{117-119}$

\section{Conclusions and future perspectives}

The last years have seen an increasing consumer demand for natural materials that can replace synthetic ones in sectors such as pharmacy, medicine and cosmetics. ${ }^{120}$ As discussed in this review, diverse studies have indeed demonstrated that natural bio-macromolecules are valid alternatives to synthetic counterparts as far as wound management is concerned. More interestingly, the possibility to nanostructure them in form of fibres through electrospinning allows the creation of biomimetic dressings with improved bioactivity for promoting tissue regeneration. In vitro and in vivo tests on diverse wound models have highlighted the efficacy of electrospun dressings based on natural materials in stimulating cells migration and proliferation, accelerating wound closure, controlling the inflammation response and, in some cases, preventing biofilms formation. However, limitations that have been emerged are associated with: the selection of appropriate solvents or polymer additives for facilitating fibre extrusion; the necessity of cross-linked procedures for improving the mechanical resistance and controlling the degradation rate of the scaffolds.

Even if the availability of biomimetic dressings represents a step forward in the wound care sector, biomedical devices with a much higher level of structural and chemical complexity are required for addressing all the different phases of the healing cascade. For this aim, a potential strategy is the creation of 3D multiscale instructive constructs through the combination of electrospinning and additive manufacturing. These approaches are under examination in other areas of regenerative medicine. ${ }^{121-123}$ For instance, bimodal and multiphasic scaffolds with controlled porosity and surface area have been obtained by the coexistence of micro- and nanoelements, and used for enhancing cell infiltration. ${ }^{121}$ When hierarchical constructs are then coupled with cell therapies, a more homogeneous distribution of cells within the scaffold can

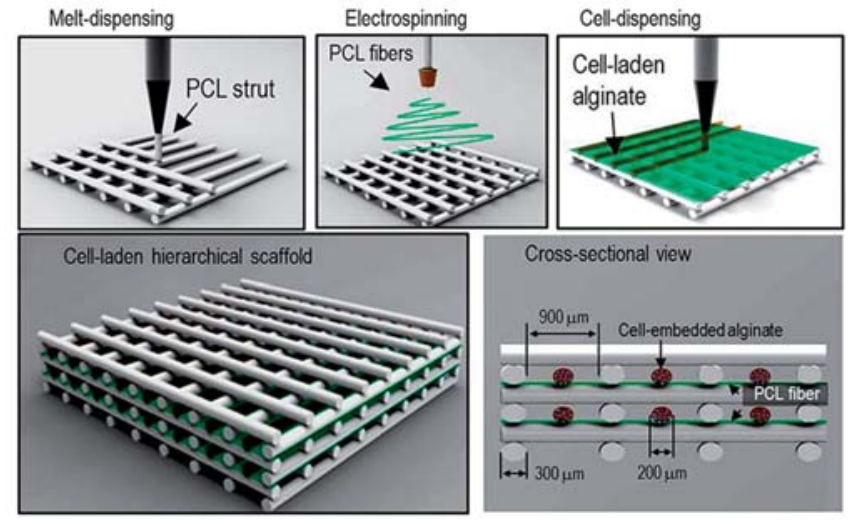

(b)

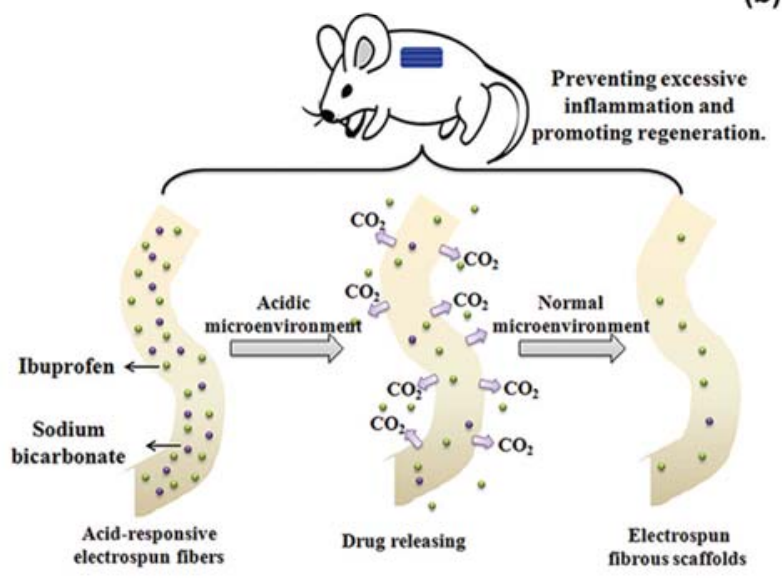

(c)

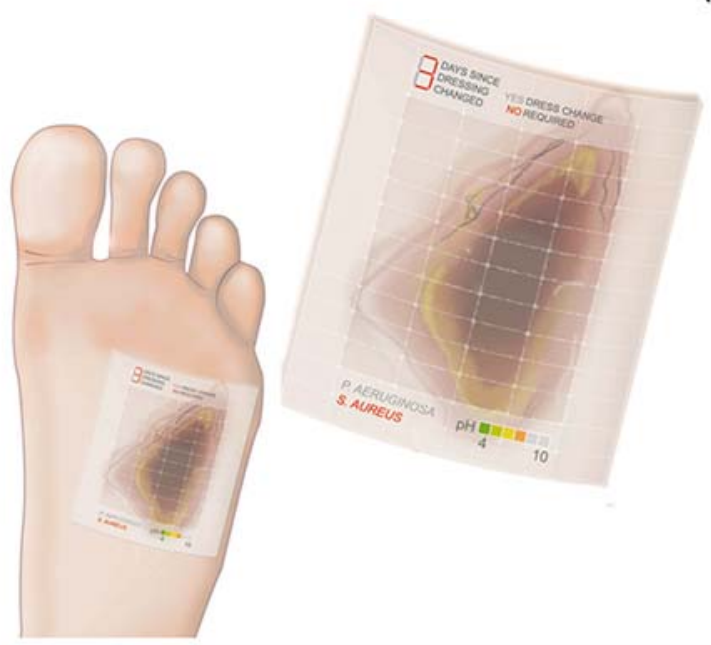

Figure 5: (a): Scheme of a manufacturing procedure leading to the production of cellladen hierarchical 3D scaffolds. The first step is the printing of PCL struts by a melting process, then PLA is electrospun in order to have interlayered micro/nanofibers. Finally cells are dispensed in a alginate matrix, obtaining cell-laden alginate struts. The crosssectional view of the final construct shows the multiscale architecture of the scaffold, where the cells are homogeneously distributed within it. Reproduced with permission from ref. 124. (b) Schematic representation of acid-responsive ibuprofen-loaded PLLA elecrospun scaffolds doped with sodium bicarbonate. The fibres release the drug at $\mathrm{pH}$ 5.0, and are able to reduce inflammation and promote tissue regeneration. Reproduced with permission from ref. 127. (c) Illustration of a future wound dressing that integrates sensors for monitoring $\mathrm{pH}$ changes and bacteria colonisation. The colour map allows gaining spatial and temporal information of the status of the wound. Reproduced with permission from ref. 125. 
be achieved, as for layers of electrospun nanofibers embedded in cell-laden alginate struts (Figure 5a). ${ }^{124}$

Together with a 3D multi-scale architecture, the achievements in the field of miniaturised sensing elements and stimuli responsive materials can be exploited in the design of dressings, which are able to sense the status of the wound and release active agents (drugs, antimicrobial compounds and growth factors) with spatial-temporal control. A review paper of T. R. Dargaville et al. has summarised how sensors integrated in a wound dressing can have diagnostic and theranostic value. ${ }^{125}$ Advantages include the detection of infections and the monitoring of temperature, $\mathrm{pH}$ and oxygen, with the purpose of reducing hospitalisation time and preventing drastic interventions, like amputations. Stimuli responsive materials, which are triggered by temperature, light or $\mathrm{pH}^{126,127}$ can be used both for sensing and for inducing changes in the properties of the scaffolds (porosity and wettability), as well as for drug delivery (Figure $5 b$ ). Figure $5 c$ shows how a patch of the future can provide real-time information on the healing process by reading $\mathrm{pH}$ changes and detecting infections.

If on one hand all the technologies needed for the development of these biomimetic, responsive and adaptive dressings are available and their integration is achievable, on the other hand regulatory and economic issues are to be taken in consideration for their realistic clinical use.

\section{References}

1 C. K. Sen, G. M. Gordillo, S. Roy, R. Kirsner, L. Lambert, T. K. Hunt, F. Gottrup, G. C. Gurtner and M. T. Longaker, Wound Rep. Reg., 2009, 17, 736-771.

2 K. Jung, S. Covington, C. K. Sen, M. Januszyk, R. S. Kirsner, G. C. Gurtner and N. H. Shah, Wound Rep. Reg., 2016, 24, 181188.

3 C. G. Decker, Y. Wang, S. J. Paluck, L. Shen, J. A. Loo, A. J. Levine, L. S. Miller and H. D. Maynard, Biomaterials, 2016, 81, 157-168.

4 R. L. Harries and K. G. Harding, Curr. Geri. Rep., 2015, 4, 265276.

5 A. Hruby and F. B. Hu, PharmacoEconomics, 2015, 33, 673689.

6 G. C. Gurtner, S. Werner, Y. Barrandon and M. T. Longaker, Nature, 2008, 453, 314-321.

7 F.-M. Chen and X. Liu, Progress Polym. Sci., 2016, 53, 86-168.

8 D. W. Green, B. Ben-Nissan, K.-S. Yoon, B. Milthorpe and H.S. Jung, J. Mater. Chem. B, 2016, 4, 2396-2406.

9 L. I.F. Moura, A. M.A. Dias, E. Carvalho and H. C. de Sousa, Acta Biomater., 2013, 9, 7093-7114.

10 J. Boateng and O. Catanzano, J. Pharm. Sci., 2015, 104, 36533680.

11 D. Tartarini and E. Mele, Front. Bioeng. Biotechnol., 2015, 3, 206.

12 M. Norouzi, S. M. Boroujeni, N. Omidvarkordshouli and M. Soleimani, Adv. Healthcare Mater., 2015, 4, 1114-1133.

13 K. A. Rieger, N. P. Birch and J. D. Schiffman, J. Mater. Chem. $B, 2013,1,4531-4541$.

14 Y.-F. Goh, I. Shakir and R. Hussain, J. Mater. Sci., 2013, 48, 3027-3054.

15 K. A. Bielefeld, S. A. Nik and B. A. Alman, Cell. Mol. Life Sci., 2013, 70, 2059-2081.
16 P. Martin, Science, 1997, 276, 75-81.

17 N. Mayet, Y. E. Choonara, P. Kumar, I. K. Tomar, C. Tyagi, L. C. Du Toit and V. Pillay, J. Pharma. Sci., 2014, 103, 2211-2230.

18 V. Falanga, Lancet, 2005, 366, 1736-1743.

19 R. Shechter and M. Schwartz, Trends Molec. Med., 2013, 19, 135-143.

20 R. A. F. Clark, K. Ghosh and m. G. Tonnesen, J. Investig. Dermat., 2007, 127, 1018-1029.

21 K. C. Broussard and J. G. Powers, Am. J. Clin. Dermatol., 2013, $14,449$.

22 D. Queen, J. H. Evans, J. d. Gaylor, J. M. Courtney and W. H. Reid, Biomaterials, 1987, 8, 372-376.

23 M. Schein, R. Saadia, J. R. Jamieson and G. A. G. Decker, British J. Surg., 1986, 73, 369-370.

24 A. Jones and D. Vaughan, J. Orthopaedic Nurs., 2005, 9, S1S11.

25 S. Thomas, Int. Wound J., 2008, 5, 602-613.

26 I. R. Sweeney, M. Miraftab and G. Collyer, Int. Wound J., 2012, 9, 601-612.

27 W. H. Eaglstein, Dermatol. Surg., 2001, 27, 175-182.

28 K. Cutting, R. White and H. Hoekstra, Int. Wound J., 2009, 6, 396-402.

29 M. Rai, A. Yadav and A. Gade, Biotechnol. Adv., 2009, 27, 7683.

30 T. Wang, X.-K. Zhu, X.-T. Xue and D.-Y. Wu, Carbohydr. Polym., 2012, 88, 75-83.

31 A. Abrigo, S. L. McArthur and P. Kingshott, Macromol. Biosci. 2014, 14, 772-792.

32 C. He, W. Nie and W. Feng, J. Mater. Chem. B, 2014, 2, 78287848.

33 G. Dan Mogosanu and A. M. Grumezescu, Int. J. Pharma., 2014, 463, 127-136.

34 R. Sridhar, R. Lakshminarayanan, K. Madhaiyan, V. A. Barathi, K. H. C. Lim and S. Ramakrishna, Chem. Soc. Rev., 2015, 44, 790-814.

35 G. Collins, J. Federici, Y. Imura and L. H. Catalani, J. Appl. Phys., 2012, 111, 044701.

36 N. Bhardwaj and S. C. Kundu, Biotechnol. Adv., 2010, 28, 325 347.

37 S. Ramakrishna, K. Fujihara, W.-E. Teo, T. Yong, Z. Ma and R. Ramaseshan, Mater. Today, 2006, 9, 40-50.

38 W. E. Teo and S. Ramakrishna, Nanotechnology, 2006, 17, R89-R106.

39 A. L. Yarin, S. Koombhongse and D. H. Reneker, J. Appl. Phys. 2001, 90, 4836-4846.

40 D. H. Reneker, A. L. Yarin, H. Fong and S. Koombhongse, J. Appl. Phys., 2000, 87, 4531-4547.

$41 \mathrm{H}$. Hajiali, M. Summa, D. Russo, A. Armirotti, V. Brunetti, R. Bertorelli, A. Athanassiou and E. Mele, J. Mater. Chem. B, 2016, 4, 1686-1695.

42 I. Liakos, L. Rizzello, H. Hajiali, V. Brunetti, R. Carzino, P. P. Pompa, A. Athanassiou, E. Mele, J. Mater. Chem. B, 2015, 3 1583-1589.

43 J. Wu, N. Wang, Y. Zhao and L. Jiang, J. Mater. Chem. A, 2013, 1, 7290-7305.

44 M. R. Abidian, D.-H. Kim and D. C. Martin, Adv. Mater., 2006, 18, 405-409.

45 H. Qu, S. Wei and Z. Guo, J. Mater. Chem. A, 2013, 1, 1151311528.

46 D. Li, Y. Wang and Y. Xia, Adv. Mater., 2004, 16, 361-366.

47 E. Mele, J. A. Heredia-Guerrero, I. S. Bayer, G. Ciofani, G. G. Genchi, L. Ceseracciu, A. Davis, E. L. Papadopoulou, M. J. Barthel, L. Marini, R. Ruffilli and A. Athanassiou, Sci. Rep., 2015, 5, 14019.

48 E. Mele, I. S. Bayer, G. Nanni, J. A. Heredia-Guerrero, R. Ruffilli, F. Ayadi, L. Marini, R. Cingolani and A. Athanassiou, Langmuir, 2014, 30, 2896-2902.

49 X. Wang, B. Ding and B. Li, Mater. Today, 2013, 16, 229-241. 
50 Z. Zhang, J. Hu and P. X. Ma, Adv. Drug Deliv. Rev., 2012, 64, 1129-1141.

51 A. Greiner and J. H. Wendorff, Angew. Chem. Int. Ed., 2007, 46, 5670-5703.

52 S.-C. Xu, C.-C. Qin, M. Yu, R.-H. Dong, X. Yan, H. Zhao, W.-P. Han, H.-D. Zhang and Y.-Z. Long, Nanoscale, 2015, 7, 1235112355.

53 R.-H. Dong, Y.-X. Jia, C.-C. Qin, Lu Zhan, X. Yan, L. Cui, Y. Zhou, X. Jiang and Y.-Z. Long, Nanoscale 2016, 8, 3482-3488.

54 X. Yan, M. Yu, L.-H. Zhang, X.-S. Jia, J.-T. Li, X.-P. Duan, C.-C. Qin, R.-H. Dong and Y.-Z. Long, Nanoscale, 2016, 8, 209-213.

55 W.-P. Han, Y.-Y. Huang, M. Yu, J.-C. Zhang, X. Yan, G.-F. Yu, H.-D. Zhang, S.-Y. Yana and Y.-Z. Long, Nanoscale, 2015, 7, 5603-5606.

56 P.-A. Mouthuy, L. Groszkowski, H. Ye, Biotechnol. Lett., 2015 37, 1107-1116.

57 P. X. Ma, Adv. Drug Deliv. Rev., 2008, 60, 184-198.

58 K. Y. Lee, L. Jeong, Y. O. Kang, S. J. Lee and W. H. Park, Adv. Drug Deliv. Rev., 2009, 61, 1020-1032.

59 L. Cen, W. Liu, L. Cui, W. Zhang and Y. Cao, Pediatric Res., 2008, 63, 492-496.

60 E. A. A. Neel, L. Bozec, J. C. Knowles, O. Syed, V. Mudera, R. Day and J. K. Hyun, Adv. Drug Deliv. Rev., 2013, 65, 429-456.

61 W. Friess, Eur. J. Pharm. Biopharm., 1998, 45, 113-136.

62 C. L. Baum and C. J. Arpey, Dermatol. Surgery, 2005, 31, 674686.

63 J. Bürck, S. Heissler, U. Geckle, M. F. Ardakani, R. Schneider A. S. Ulrich and M. Kazanci, Langmuir, 2013, 29, 1562-1572.

64 B. Dong, O. Arnoult, M. E. Smith and G. E. Wnek, Macromol. Rapid Commun. 2009, 30, 539-542.

65 M. J. Fullana and G. E. Wnek, Drug Deliv. and Transl. Res., 2012, 2, 313-322.

66 L. Meng, O. Arnoult, M. Smith and G. E. Wnek, J. Mater. Chem., 2012, 22, 19412-19417.

67 S. Torres-Giner, J. V. Gimeno-Alcaniz, M. J. Ocio and J. M. Lagaron, ACS Appl. Mater. Interf. 2009, 1, 218-223.

68 K. S. Rho, L. Jeong, G. Lee, B.-M. Seo, Y. J. Park, S.-D. Hong, S. Roh, J. J. Cho, W. H. Park and B.-M. Min, Biomaterials, 2006, 27, 1452-1461.

69 H. M. Powell, D. M. Supp and S. T. Boyce, Biomaterials, 2008, 29, 834-843.

70 J. R. Venugopal, Y. Zhang and S. Ramakrishna, Artif. Organs, 2006, 30, 440-446.

71 M. Gümüşderelioğlu, S. Dalkıranoğlu, R. Seda Tığlı Aydın and S. Çakmak, J. Biomed. Mater. Res. Part A, 2001, 98A, 461472.

72 C. Huang, X. Fu, J. Liu, Y. Qi, S. Li and H. Wang, Biomaterials, 2012, 33, 1791-1800

73 C. H. Lee, S. H. Chang, W. J. Chen, K. C. Hung, Y. H. Lin, S. J. Liu, M. J. Hsieh, J. H. Pang and J. H. Juang, J. Colloid. Interface Sci., 2015, 439, 88-97.

74 Z.-M. Huang, Y. Z. Zhang, S. Ramakrishna and C. T. Lim, Polymer, 2004, 45, 5361-5368.

75 M. Erencia, F. Cano, J. A. Tornero, M. M. Fernandes, T. Tzanov, J. Macanás and F. Carrillo, J. Appl. Polym. Sci., 2015, 132, 42115 .

76 P. Rujitanaroj, N. Pimpha, P. Supaphol, Polymer, 2008, 49, 4723-4732.

77 A. A. Dongargaonkar, G. L. Bowlin and H. Yang, Biomacromol., 2013, 14, 4038-4045.

78 C.-H. Yao, J.-Y. Yeh, Y.-S. Chen, M.-H. Li and C.-H. Huang, J. Tissue Eng. Regen. Med., 2015.

79 E. J. Chong, T. T. Phan, I. J. Lim, Y. Z. Zhang, B. H. Bay, S. Ramakrishna and C. T. Lim, Acta Biomater., 2007, 3, 321330

80 S. E. Kim, D. N. Heo, J. B. Lee, J. R. Kim, S. H. Park, S. H. Jeon and I. K. Kwon, Biomed. Mater., 2009, 4, 044106.
81 S.-Y. Gu, Z.-M. Wang, J. Ren and C.-Y. Zhang, Mater. Sci. Eng. C, 2009, 29, 1822-1828.

82 G. Kogan, L. Soltes, R. Stern and P. Gemeiner, Biotechnol. Lett., 2007, 29, 17-25.

83 W. Y. J. Chen and G. Abatangelo, Wound Rep. Reg., 1999, 7, 79-89.

84 G. D. Prestwich, J. Control. Release, 2011, 155, 193-199.

85 R. Uppal, G. N. Ramaswamy, C. Arnold, R. Goodband and Y. Wang, J. Biomed. Mater. Res. Part B: Appl. Biomater., 2011, 97, 20.

86 Y. Ji, K. Ghosh, X. Zheng Shu, B. Li, J. C. Sokolov, G. D. Prestwich, R. A. F. Clark and M. H. Rafailovic, Biomaterials, 2006, 27, 3782-3792.

87 Y. Liu, G. Ma, D. Fang, J. Xu, H. Zhang and J. Nie, Carbohydr. Polym., 2011, 83, 1011-1015.

88 Y. Qian, L. Li, C. Jiang, W. Xu, Y. LV, L. Zhong, K. Cai and L. Yang, Int. J. Biolog. Macromol. 2015, 79, 133-143.

89 F.-Y. Hsu, Y.-S. Hung, H.-M. Liou and C.-H. Shen, Acta Biomater., 2010, 6, 2140-2147.

90 N. Laurens, P. Koolwijk and M. P. M. De Maat, J. Thrombos. Haemostas., 2006, 4, 932-939.

91 G. E. Wnek, M. E. Carr, D. G. Simpson and G. L. Bowlin, Nano Lett., 2003, 3, 213-216.

92 S. Baker, J. Sigley, C. C. Helms, J. Stitzel, J. Berry, K. Bonin and M. Guthold, Mater. Sci. Eng. C, 2012, 32, 215-221.

93 M. C. McManus, E. D. Boland, H. P. Koo, C. P. Barnes, K. J. Pawlowski, G. E. Wnek, D. G. Simpson and G. L. Bowlin, Acta Biomater., 2006, 2, 19-28.

94 S. W. Rothwell, E. Sawyer, J. Dorsey, W. S. Flournoy, T. Settle, D. Simpson, G. Cadd, P. Janmey, C. White and K. A. Szabo, J. Mater. Sci.: Mater. Med., 2009, 20, 2155-2166.

95 N. M. Alves and J. F. Mano, Int. J. Biolog. Macromol., 2008, 43, 401-414.

96 R. Jayakumar, M. Prabaharan, S. V. Nair and H. Tamura, Biotech. Adv., 2010, 28, 142-150.

97 K. A. Rieger and J. D. Schiffman, Carbohydr. Polym., 2014 113, 561-568.

98 N. Charernsriwilaiwat, T. Rojanarata, T. Ngawhirunpat, M. Sukma and P. Opanasopit, Int. J. Pharma., 2013, 452, 333343.

99 W. A. Sarhan, H. M. E. Azzazy and I. M. El-Sherbiny, ACS Appl. Mater. Interf., 2016, 8, 6379-6390.

100 W. A. Sarhan and H. M. E. Azzazy, Carbohydr. Polym., 2015, 122, 135-143.

101 B. Veleirinho, D. S. Coelho, P. F. Dias, M. Maraschin, R. M. Ribeiro-do-Valle and J. A. Lopes-da-Silva, Int. J. Biolog. Macromol., 2012, 51, 343-350.

102 S. D. Sarkar, B. L. Farrugia, T. R. Dargaville and S. Dhara, J. Biomed. Mater. Res. Part A, 2013, 101, 3482-3492.

103 F. Khan and S. R. Ahmad, Macromol. Biosci., 2013, 13, 395-421.

104 N. Bhattarai, Z. Li, D. Edmondson and M. Zhang, Adv. Mater., 2006, 18, 1463-1467.

105 N. Bhattarai and M. Zhang, Nanotechnology, 2007, 18, 455601.

106 Y. J. Kim, K. J. Yoon and S. W. Ko, J. Appl. Polym. Sci., 2000, 78, 1797-1804.

107 H. Hajiali, J. A. Heredia-Guerrero, I. Liakos, A. Athanassiou and E. Mele, Biomacromol., 2015, 16, 936-943.

108 K. T. Shalumon, K. H. Anulekha, S. V. Nair, S. V. Nair, K. P. Chennazhi and R. Jayakumar, Int. J. Biolog. Macromol., 2011, 49, 247-254.

109 C. E. Pegg, G. H. Jones, T. J. Athauda, R. R. Ozer and J. M. Chalker, Chem. Commun., 2014, 50, 156-158.

110 K. Kataria, A. Gupta, G. Rath, R. B. Mathur and S. R. Dhakate, Int. J. Pharma., 2014, 469, 102-110. 
111 G. H. Altman, F. Diaz, C. Jakuba, T. Calabro, R. L. Horan, J. Chen, H. Lu, J. Richmond and D. L. Kaplan, Biomater., 2003, 24, 401-416.

112 S. Lin, M. Chen, H. Jiang, L. Fan, B. Sun, F. Yu, X. Yang, X. Lou, C. He and H. Wang, Coll. Surf. B: Biointerf., 2016, 139, 156-163.

113 B.-M. Min, G. Lee, S. H. Kim, Y. S. Nam, T. S. Lee and W. H. Park, Biomater., 2004, 25, 1289-1297.

114 H. W. Ju, O. J. Lee, J. M. Lee, B. M. Moon, H. J. Park, Y. R. Park, M. C. Lee, S. H. Kim, J. R. Chao, C. S. Ki and C. H. Park, Int. J. Biolog. Macromol., 2016, 85, 29-39.

115 Y.-H. Shan, L.-H. Peng, X. Liu, X. Chen, J. Xiong and J.-Q. Gao, Int. J. Pharma., 2015, 479, 291-301.

116 L. Fan, H. Wang, K. Zhang, Z. Cai, C. He, X. Sheng and X. Mo, RSC Adv., 2012, 2, 4110-4119.

117 S. Shahverdi, M. Hajimiri, M. A. Esfandiari, B. Larijani, F. Atyabi, A. Rajabiani, A. R. Dehpour, A. A. Gharehaghaji and R. Dinarvand, Int. J. Pharma., 2014, 473, 345-355.

118 S. Guang, Y. An, F. Ke, D. Zhao, Y. Shen and H. Xu, J. Appl. Polym. Sci., 2015, 132, 42503.

119 S. Guzman-Puyol, J. A. Heredia-Guerrero, L. C., H. Hajiali, C. Canale, A. Scarpellini, R. Cingolani, I. S. Bayer, A. Athanassiou and E. Mele, ACS Biomater. Sci. Eng., 2016.

120 F. Reyes-Jurado, A. Franco-Vega, N. Ramirez-Corona, E. Palou and A. Lopez-Malo, Food Eng. Rev., 2015, 7, 275.

121 P. D. Dalton, C. Vaquette, B. L. Farrugia, T. R. Dargaville, T. D. Brown and D. W. Hutmacher, Biomater. Sci., 2013, 1, 171-185.

122 S. M. Oliveira, R. L. Reis and J. F. Mano, Biotech. Adv., 2015, 33, 842-855.

123 S. M. Giannitelli, P. Mozetic, M. Trombetta and A. Rainer, Acta Biomater., 2015, 24, 1-11.

124 M. G. Yeo and G. H. Kim, J. Mater. Chem. B, 2014, 2, 314 324.

125 T. R. Dargaville, B. L. Farrugia, J. A. Broadbent, S. Pace, Z. Upton and N. H. Voelcker, Biosens. Bioelectr., 2013, 41, 3042.

126 S. Shy Liow, Q. Dou, D. Kai, A. A. Karim, K. Zhang, F. Xu and X. J. Loh, ACS Biomater. Sci. Eng., 2016, 2, 295-316.

127 Z. Yuan, J. Zhao, W. Zhu, Z. Yang, B. Li, H. Yang, Q. Zheng and W. Cui, Biomater. Sci., 2014, 2, 502-511. 